\title{
UTILIZAÇÃO DE MÉTODOS COMBINADOS PARA OBTENÇÃO DE MAMÃO FORMOSA (Carica papaya) SECO
}

\author{
CAROLINA CASTILHO GARCIA* \\ DIEGO CANIZARES** \\ KEILA DE SOUZA SILVA*** \\ RÓGER DARROS-BARBOSA****

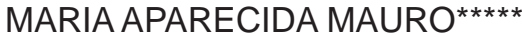

\begin{abstract}
O objetivo do presente trabalho foi avaliar a influência do branqueamento em água quente, da desidratação osmótica em solução de sacarose e da aplicação de cobertura comestível de pectina na cor e atividade de água de fatias de mamão Formosa, após secagem convectiva. Nos experimentos iniciais, com base em planejamento fatorial, avaliou-se a influência da concentração da solução desidratante e do tempo de desidratação osmótica na cor e atividade de água de fatias frescas e branqueadas de mamão. Não foram verificadas alterações significativas na cor das amostras nos intervalos estudados. Encontrou-se expressão para a atividade de água das frutas branqueadas desidratadas osmoticamente. Posteriormente avaliou-se a influência do branqueamento, da desidratação osmótica, da aplicação de cobertura comestível e de combinações entre esses pré-tratamentos na cor e atividade de água das fatias de mamão após secagem convectiva. Verificou-se que o branqueamento não representou pré-tratamento vantajoso para a manutenção da cor e para a redução da atividade de água das frutas secas. As frutas frescas desidratadas osmoticamente com ou sem aplicação de cobertura comestível apresentaram os melhores resultados.
\end{abstract}

PALAVRAS-CHAVES: DESIDRATAÇÃO OSMÓTICA; BRANQUEAMENTO; COBERTURA COMESTÍVEL; MAMÃO - SECAGEM.

\footnotetext{
* Professora, Doutora em Engenharia e Ciência de Alimentos, Núcleo de Alimentos, Universidade Tecnológica Federal do Paraná, Medianeira, PR (e-mail: carolinacgarcia@utfpr.edu.br).

** Doutoranda em Engenharia e Ciência de Alimentos, Departamento de Engenharia e Tecnologia de Alimentos, Universidade Estadual Paulista (UNESP), São José do Rio Preto, SP (e-mail: keilasouzas@ yahoo.com.br).

*** Mestrando em Engenharia e Ciência de Alimentos, Departamento de Engenharia e Tecnologia de Alimentos, UNESP, São José do Rio Preto, SP (e-mail: canizares-diego@hotmail.com).

**** Professor, Doutor em Food Science emphasis in Food Engineering, Departamento de Engenharia e Tecnologia de Alimentos, UNESP, São José do Rio Preto, SP (e-mail: roger@ibilce.unesp.br).

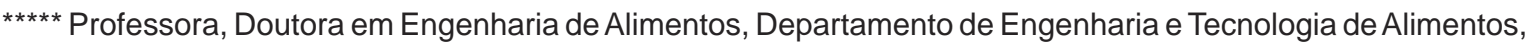
UNESP, São José do Rio Preto, SP (e-mail: cidam@ibilce.unesp.br).
} 


\section{INTRODUÇÃo}

O mamão, fruta proveniente do Sul do México, é típica de regiões de clima tropical. Quando maduro, sua polpa apresenta coloração variando do amarelo ao laranja-avermelhado e sabor doce e delicado. Trata-se de fruta nutritiva que apresenta quantidades apreciáveis de vitaminas A e C, além de minerais como, ferro, cálcio e fósforo. Os carotenoides majoritários da polpa do mamão são o licopeno, a $\beta$-criptoxantina e o $\beta$-caroteno, pigmentos tipicamente vermelho-alaranjados (KIMURA, RODRIGUEZ-AMAYA e YOKOYAMA, 1991).

Devido à perecibilidade do mamão, seu processamento representa alternativa para diminuir as altas perdas na colheita e pós-colheita da fruta, agregando valor ao produto final.

A desidratação osmótica (DO) envolve a imersão de determinado produto em solução aquosa hipertônica, resultando em decréscimo da umidade com incremento simultâneo de sólidos, além de mudanças na composição química dos produtos desidratados (EREN e KAYMAKERTEKIN, 2007; FERNANDES et al., 2006; SERENO, MOREIRA e MARTINEZ, 2001). Pode-se modificar a formulação do alimento, durante a DO, mediante incorporação de solutos na estrutura porosa de frutas e vegetais (TORREGIANI e BERTOLO, 2001; FITO et al., 2001). A adição de cálcio nas soluções desidratantes vem recebendo grande atenção devido aos efeitos desejáveis na manutenção da firmeza dos tecidos, como constatado em tomate cereja e mamão (HEREDIA, BARRERA e ANDRÉS, 2007; RODRIGUES, BARRERA e ANDRÉS, 2003).

$O$ branqueamento, amplamente difundido na preparação de alimentos minimamente processados, objetiva a inativação enzimática, destruição/injúria de micro-organismos para reduzir a carga microbiana inicial do alimento ou sensibilização para posterior tratamento dos microorganismos sobreviventes (ALZAMORA et al., 1997). Os inconvenientes desse processamento térmico são os danos causados ao tecido vegetal, os quais resultam em seu amolecimento (DOYMAZ, 2007; KOWALSKA, LENART e LESZCZYK, 2008; ESCOBAR et al., 2007; ALZAMORA et al., 1997). Quando aplicado previamente à secagem, ou outro método de processamento de alimentos, verificam-se maiores taxas de desidratação e/ou menores tempos de tratamento para determinada umidade final (DOYMAZ, 2007; KOWALSKA, LENART e LESZCZYK, 2008; ESCOBAR et al., 2007).

As coberturas comestíveis são finas camadas de material comestível aplicadas à superfície dos alimentos para criar barreira seletiva ao transporte de gases (VARGAS et al., 2008). As coberturas de polissacarídeos apresentam boas propriedades de barreira ao oxigênio, particularmente em condições de baixa umidade (CUQ, GINTARD e GUILBERT, 1995), sendo adequadas como compostos básicos para a formulação de revestimentos comestíveis aplicados previamente à secagem.

A secagem, processo térmico complexo porque ocorre simultaneamente transferência de massa e de calor, visa a remoção da água do alimento para níveis que minimizem ou inibam o crescimento microbiano e as reações de deterioração (DOYMAZ, 2007). O efeito da secagem nas características sensoriais e nutricionais do produto final e a utilização de pré-tratamentos como alternativa para melhorar essas características vêm sendo estudado por diversos pesquisadores (LEERATANARAK, DEVAHASTIN e CHIEWCHAN, 2006; ZHAO e CHANG, 1995).

A proposta do presente trabalho foi avaliar a influência de pré-tratamentos na cor e atividade de água de fatias de mamão após secagem convectiva. Os experimentos foram divididos em duas etapas, sendo avaliada inicialmente a influência da concentração da solução e do tempo de desidratação osmótica em fatias frescas e branqueadas de mamão Formosa, imersas em soluções de sacarose acrescidas de cálcio e ácido lático sobre sua cor e atividade de água. Posteriormente avaliou-se a influência de pré-tratamentos (branqueamento, aplicação de cobertura comestível, desidratação osmótica e combinações entre eles) sobre alterações na cor e redução da atividade de água das frutas após secagem convectiva. 


\section{MATERIAL E MÉTODOS}

\subsection{PREPARO DAS AMOSTRAS}

Mamões do cultivar Formosa, provenientes do estado de São Paulo (Brasil), foram adquiridos na Companhia de Entrepostos e Armazéns Gerais de São Paulo, localizada em São José do Rio Preto (CEAGESP). Frutas maduras com casca alaranjada e tendo, aproximadamente, $8,89 \% \pm 0,73$ de açúcares redutores foram utilizadas nos experimentos.

Os mamões foram cortados longitudinalmente em quatro, sendo as sementes retiradas e cada parte seccionada com cortador manual em fatias de $3,6 \mathrm{~cm}$ de diâmetro. Os cilindros de mamão foram cortados em fatias de, aproximadamente, $1,0 \mathrm{~cm}$ de espessura. As amostras foram armazenadas e misturadas em saco plástico do qual, aleatoriamente, retiraram-se os pedaços para os experimentos.

\subsection{BRANQUEAMENTO}

As fatias de mamão foram dispostas em cesto de material inoxidável e mergulhadas em água fervente $\left(98,3^{\circ} \mathrm{C}\right)$ por 2 minutos. Após esse tempo, as amostras foram resfriadas em água corrente por 1 min para serem utilizadas nos experimentos.

\subsection{DESIDRATAÇÃO OSMÓTICA}

Prepararam-se as soluções osmóticas com sacarose comercial (açúcar refinado), lactato de cálcio pentahidratado grau alimentício em pó (PURAC Synthesis - Brasil), L (+)-ácido lático grau alimentício em solução a 85\% (PURAC Synthesis - Brasil) e água destilada.

Para as frutas frescas e branqueadas foram preparadas soluções nas concentrações de $40 \%, 50 \%$ e $60 \% \mathrm{p} / \mathrm{p}$ de sacarose, adicionadas de $1 \% \mathrm{p} / \mathrm{p}$ de lactato de cálcio e $0,1 \mathrm{M}$ de ácido lático. Utilizaram-se os tempos de desidratação de 60, 120 e 180 min para as fatias de fruta in natura e de 30, 105 e 180 min para as branqueadas. Aumentou-se o intervalo de tempo dos experimentos com as frutas branqueadas de maneira a encontrar diferenças significativas entre as respostas.

Os ensaios de desidratração osmótica (DO) foram conduzidos em cuba de aço inoxidável com camisa externa de circulação de água a $27^{\circ} \mathrm{C}$, bombeada a partir de banho ultratermostatizado. Mantiveram-se as soluções sob agitação constante de 1000 rpm, conduzida por agitador mecânico. Adotou-se a relação entre massa de amostra e massa de solução de, no mínimo, 1:10. Após os tempos de processamento, as fatias foram retiradas das soluções, lavadas em água destilada por $10 \mathrm{~s}$ e secas em papel absorvente para eliminar o excesso de solução na superfície das amostras (GARCIA, MAURO e KIMURA, 2007).

As frutas frescas e branqueadas foram desidratadas osmoticamente, previamente à secagem, utilizando-se as condições estabelecidas na primeira etapa dos experimentos. Assim, as frutas frescas foram pré-desidratadas em solução de sacarose a $50 \% \mathrm{p} / \mathrm{p}$, adicionada de $1 \% \mathrm{p} / \mathrm{p}$ de lactato de cálcio e 0,1 M de ácido lático durante $12 \mathrm{~min}$. Os mamões branqueados foram prédesidratados em solução de sacarose a $50 \% \mathrm{p} / \mathrm{p}$, adicionada de $1 \% \mathrm{p} / \mathrm{p}$ de lactato de cálcio e $0,1 \mathrm{M}$ de ácido lático por 105 min.

\subsection{COBERTURA COMESTÍVEL}

Utilizou-se pectina amidada de baixa metoxilação GRINDSTED ${ }^{\circledR}$ LA 210 (grau de metoxilação: 0,34; grau de amidação: 0,17 da Danisco - Brasil) para cobrir as fatias de mamão. A solução de pectina a $2 \% \mathrm{p} / \mathrm{p}$, preparada a $70^{\circ} \mathrm{C}$, foi aplicada na superfície das amostras a $40^{\circ} \mathrm{C}$ por imersão durante $1 \mathrm{~min}$. Ativou-se a gelificação da cobertura mediante imersão das fatias em 
solução de lactato de cálcio pentahidratado em pó (PURAC Synthesis - Brasil) a 2,8\% p/p por $30 \mathrm{~s}$. Removeu-se o excesso de cobertura, lavando as amostras por imersão em água destilada durante $30 \mathrm{~s}$.

\subsection{SECAGEM}

Mamões frescos e branqueados, desidratados osmoticamente ou não, cobertos ou não, foram secos em secador convectivo com velocidade do ar de $1,0 \mathrm{~m} \cdot \mathrm{s}^{-1}$ a $70^{\circ} \mathrm{C}$ por $240 \mathrm{~min}$. Realizouse a rotação das bandejas dentro do secador a cada $30 \mathrm{~min}$.

Dois lotes de amostras foram processados. No primeiro, frutas frescas e branqueadas, desidratadas osmoticamente ou não (F, B, F-DO e B-DO) foram secas em paralelo em duplicata. No segundo lote, as amostras foram cobertas com pectina antes da secagem (F-CO, B-CO, F-DO-CO e B-DO-CO), também realizada em duplicata.

\subsection{METODOLOGIAS ANALÍTICAS}

A atividade de água e a cor das amostras foram avaliadas antes e após os processamentos. Mediu-se a atividade de água em triplicata a $25^{\circ} \mathrm{C}$ em higrômetro AW SPRINT (NOVASINA, 1999). A primeira etapa de experimentos foi realizada no primeiro semestre de 2007. Nessa época, o espectrofotômetro Colorflex (HunterLab, USA) ainda não havia sido adquirido e, portanto, a cor das amostras foi medida em quadruplicata por capturador de imagem com câmera digital instalada em cabine de luz apropriada e software acadêmico (V-01 LensEye) para análise de cor, conforme metodologia proposta por Luzuriaga, Devahastin e Chiewchan (1997). Utilizaram-se os padrões $L^{*}=71,941 ; a^{*}=36,067$ e $b^{*}=67,857$. A segunda etapa de experimentos foi realizada no primeiro semestre de 2008, quando o espectrofotômetro Colorflex (HunterLab, EUA) já havia sido adquirido. Assim, avaliou-se a cor dessas amostras no referido equipamento, utilizando-se software Universal versão $4.10 \mathrm{com}$ as seguintes configurações: iluminante D65, observador a $10^{\circ}$ e leitura dos valores absolutos de $L^{*}$, $a{ }^{*}$ e b* (HUNTERLAB, 2007).

\subsection{DELINEAMENTO EXPERIMENTAL E MODELAGEM}

Adotou-se planejamento fatorial $2^{2}$ com 3 pontos centrais para delinear os ensaios de DO e para estimar os efeitos principais das variáveis de processo (concentração da solução osmótica e tempo de processamento - variáveis independentes) na atividade de água e cor das fatias de mamão frescas e branqueadas (variáveis dependentes ou respostas). Realizou-se cada experimento com diferentes amostras in natura, de modo que as respostas representassem a razão entre o valor para a fruta processada e o valor para a fresca.

Os coeficientes de regressão foram utilizados para derivar modelo matemático linear (Equação 1). A análise de variância permitiu a identificação das variáveis codificadas, cujos efeitos nas respostas de interesse foram significativos ao nível de confiança de $95 \%(p<0,05)$ (BARROS NETO, SCARMINIO e BRUNS, 2002):

$$
\mathrm{Y}=\beta_{\mathrm{k} 0}+\sum_{i=1}^{2} \beta_{k i} x_{i}+\sum_{i=1}^{1} \sum_{j=i+1}^{2} \beta_{k i j} x_{i} x_{j}
$$

em que:

$\beta_{k 0}, \beta_{k i}$ e $\beta_{k i j}=$ coeficientes (constantes);

$x=$ fatores ou variáveis independentes; $\mathrm{e}$

$Y=$ respostas ou variáveis dependentes. 
Os valores codificados utilizados na Equação 1 foram obtidos a partir das variáveis não codificadas, mediante as seguintes expressões:

$$
\begin{aligned}
& x_{1}=(C-50) / 10 \\
& x_{2}^{F}=(t-120) / 60 \\
& x_{2}^{B}=(t-105) / 75
\end{aligned}
$$

em que:

$C=$ concentração da solução osmótica (\% p/p);

$t=$ tempo de processamento ( $\mathrm{min})$.

O sobrescrito $F$ foi utilizado para indicar os experimentos realizados com as frutas frescas e o $B$ com as branqueadas.

Todas as análises estatísticas foram realizadas usando-se o programa Statistica 7.0 (STATSOFT, 2004), sendo a ordem dos experimentos definida aleatoriamente.

\section{RESULTADOS E DISCUSSÃO}

\subsection{AVALIAÇÃO DAS CONDIÇÕES DE DESIDRATAÇÃO OSMÓTICA DAS FRUTAS FRESCAS}

A Tabela 1 apresenta o delineamento fatorial, as variáveis codificadas e as respostas obtidas nos experimentos de DO com as frutas frescas.

\begin{tabular}{|c|c|c|c|c|c|c|c|c|}
\hline \multirow[b]{2}{*}{ Exp. } & \multicolumn{2}{|c|}{$\begin{array}{c}\text { Variáveis não } \\
\text { codificadas }\end{array}$} & \multicolumn{2}{|c|}{$\begin{array}{l}\text { Variáveis } \\
\text { codificadas }\end{array}$} & \multicolumn{4}{|c|}{ Respostas } \\
\hline & $\begin{array}{l}\text { Conc. } \\
(\%)\end{array}$ & $\begin{array}{l}\text { Tempo } \\
\text { (min) }\end{array}$ & $x_{1}$ & $x_{2}^{F}$ & $\frac{a w_{F-D O}}{a w_{F}}$ & $\frac{L_{F-D O}^{*}}{L_{F}^{*}}$ & $\frac{b_{F-D O}^{*}}{b_{F}^{*}}$ & $\frac{a_{F-D O}^{*}}{a_{F}^{*}}$ \\
\hline 1 & 40 & 60 & -1 & -1 & 0,987 & 0,90 & 1,01 & 0,91 \\
\hline 2 & 60 & 60 & +1 & -1 & 0,988 & 1,00 & 0,99 & 0,98 \\
\hline 3 & 40 & 180 & -1 & +1 & 0,981 & 1,04 & 1,01 & 1,05 \\
\hline 4 & 60 & 180 & +1 & +1 & 0,977 & 1,00 & 1,01 & 1,00 \\
\hline 5 & 50 & 120 & 0 & 0 & 0,989 & 0,86 & 1,01 & 0,84 \\
\hline 6 & 50 & 120 & 0 & 0 & 0,990 & 1,00 & 0,98 & 0,88 \\
\hline 7 & 50 & 120 & 0 & 0 & 0,983 & 1,03 & 0,96 & 1,02 \\
\hline
\end{tabular}

\section{TABELA 1 - DELINEAMENTO EXPERIMENTAL E RESPOSTAS OBTIDAS NOS ENSAIOS DE DESIDRATAÇÃO OSMÓTICA COM AS FRUTAS FRESCAS}

A atividade de água média e os parâmetros médios $L^{*}$, $a^{*}$ e $b^{*}$ foram $0,990 \pm 0,000$; $36,57 \pm 4,98 ; 38,15 \pm 0,72$ e $31,80 \pm 3,65$, respectivamente. As amostras frescas desidratadas em solução $60 \%$ por 3 horas apresentaram a menor atividade de água $(0,968)$ e a menor relação entre a atividade de água das frutas processadas e das frescas (Tabela 1). 
A análise de variância das relações $a w_{F-D O} / a w_{F}, L_{F-D O}^{*} / L_{F}^{*}, a_{F-D O}^{*} / a_{F}^{*}$ e $b_{F-D O}^{*} / b_{F}^{*}$ mostrou que nenhum termo do modelo linear foi estatisticamente significativo ao nível de $95 \%$ de confiança. Os modelos codificados que representariam o comportamento dessas respostas durante o tratamento osmótico não evidenciaram bom ajuste, apresentando coeficientes de determinação de 0,$5557 ; 0,3906 ; 0,1368$ e 0,3007 , respectivamente.

Os valores de $L^{*}, a^{*} e b^{*}$ da maioria das frutas frescas desidratadas osmoticamente aproximaram-se dos obtidos para as frutas frescas, uma vez que as razões (Tabela 1) ficaram próximas de 1,00 .

Os parâmetros $a^{*} e b^{*}$ indicam a intensidade da cor, sendo que $a^{*}$ varia do vermelho (valores positivos) ao azul (valores negativos) e $b^{*}$ do amarelo (valores positivos) ao verde (valores negativos). Tendo em vista que o conteúdo de carotenoides está vinculado à cor, os resultados sugeriram ter ocorrido considerável retenção dos carotenoides durante a DO. Isso se deve ao fato das amostras ficarem imersas na solução desidratante e também à temperatura amena utilizada no processamento $\left(27^{\circ} \mathrm{C}\right)$ (TONON, BARONI e HUBINGER, 2007; SHI et al., 1999; HENG, GUILBERT e CUQ, 1990).

\subsection{AVALIAÇÃO DAS CONDIÇÕES DE DESIDRATAÇÃO OSMÓTICA DAS FRUTAS BRANQUEADAS}

A Tabela 2 apresenta o delineamento fatorial, as variáveis codificadas e as respostas obtidas nos experimentos de DO com as frutas branqueadas.

\section{TABELA 2 - DELINEAMENTO EXPERIMENTAL E RESPOSTAS OBTIDAS NOS ENSAIOS DE DESIDRATAÇÃO OSMÓTICA COM AS FRUTAS BRANQUEADAS}

\begin{tabular}{ccccccccc}
\hline & \multicolumn{1}{c}{ Variáveis não codificadas } & \multicolumn{2}{l}{$\begin{array}{l}\text { Variáveis } \\
\text { codificadas }\end{array}$} \\
& & & & & & & \\
Exp. & Conc. $(\% \mathrm{p} / \mathrm{p})$ & Tempostas & \\
& & & $x_{1}$ & $x_{2}^{B}$ & $\frac{a w_{B-D O}}{a w_{F}}$ & $\frac{L_{B-D O}^{*}}{L_{F}^{*}}$ & $\frac{a_{B-D O}^{*}}{a_{F}^{*}}$ & $\frac{b_{B-D O}^{*}}{b_{F}^{*}}$ \\
\hline 1 & 40 & 30 & -1 & -1 & 0,995 & 0,95 & 1,22 & 1,04 \\
2 & 60 & 30 & +1 & -1 & 0,994 & 1,03 & 1,18 & 1,07 \\
3 & 40 & 180 & -1 & +1 & 0,986 & 1,03 & 1,21 & 1.11 \\
4 & 60 & 180 & +1 & +1 & 0,971 & 0,96 & 1,32 & 1,01 \\
5 & 50 & 105 & 0 & 0 & 0,988 & 1,09 & 1,28 & 1,19 \\
6 & 50 & 105 & 0 & 0 & 0,988 & 0,94 & 1,31 & 1,00 \\
7 & 50 & 105 & 0 & 0 & 0,987 & 0,99 & 1,00 & 1,08 \\
\hline
\end{tabular}

A atividade de água média e os parâmetros médios de $L^{*}$, $a^{*}$ e $b^{*}$ foram $0,989 \pm 0,001$; $41,61 \pm 1,56 ; 36,52 \pm 2,02$ e 39,61 $\pm 1,86$, respectivamente. Os valores da atividade de água e do parâmetro $b^{*}$ mostraram-se similares aos encontrados nos experimentos com as frutas frescas (Tabelas 1 e 2). A atividade de água média das frutas branqueadas $(0,990 \pm 0,000)$ não diferiu significativamente da obtida para as frutas frescas $(0,989 \pm 0,001)$ com $5 \%$ de significância (Tabela 2).

As atividades de água das amostras branqueadas e desidratadas e das frescas desidratadas osmoticamente nas mesmas condições de processo resultaram muito próximas (Tabelas 1 e 2).

Todos os termos do modelo linear da relação $a w_{B-O D} / a w_{F}$ foram significativos ao nível de 
95\% de confiança. A Equação 5 representa esse modelo:

$$
Y=0,987-0,004 x_{1}-0,008 x_{2}^{B}-0,003 x_{1} x_{2}^{B}
$$

em que:

$Y=$ relação entre atividade de água das amostras branqueadas e submetidas à $\mathrm{DO}$ e das frescas $\left(a w_{B-O D} / a w_{F}\right)$

$x_{1}=$ variável concentração da solução osmótica $(\%$ p/p);

$x_{2}^{B}=$ variável tempo de processamento $(\min ) ; \mathrm{e}$

$x_{1} x_{2}^{B}=$ termo de interação entre as variáveis independentes.

A Equação 5 apresentou bom ajuste aos dados experimentais, com coeficiente de determinação de 0,9945. Ambas as variáveis afetaram negativamente a resposta, mostrando que menores valores de atividade de água estão correlacionados a maiores concentrações de solução desidratante e tempo de DO. O tempo de processamento apresentou maior influência negativa na resposta $a w_{B-O D} / a w_{F}$ do que a concentração da solução osmótica.

Chan e Kwok (1975) verificaram que a atividade da enzima invertase em mamões do cultivar Solo foi de $0,821 \mathrm{U} \cdot \mathrm{mg}^{-1}$ de proteína. Após sua manipulação (remoção da casca e sementes, corte e trituração), metade do conteúdo de sacarose presente nas frutas seria convertida em açúcares redutores após 2,66 min, caso a enzima não fosse inativada. No presente trabalho, a atividade da invertase pode ter criado fluxos que desestabilizaram o sistema. Assim, a sacarose transferida da solução osmótica para o mamão deve ter sido convertida enzimaticamente a açúcares redutores. As frutas branqueadas, por outro lado, apresentaram composição de açúcares mais estável durante a DO, o que deve ter contribuído para o ajuste dos dados experimentais a uma equação.

O modelo experimental que descreveria o comportamento das razões de $L_{B-O D}^{*} / L_{F}^{*}$, $a_{B-O D}^{*} / a_{F}^{*}$ e $b_{B-O D}^{*} / b_{F}^{*}$ durante os tratamentos osmóticos não apresentou bom ajuste, com coeficientes de determinação de 0,3141; 0,1541 e 0,2195, respectivamente.

As frutas branqueadas submetidas à DO apresentaram maior intensificação da cor que os mamões frescos, sendo os valores de $a^{*}$ os mais afetados pelo tratamento osmótico (Tabela 2). As amostras processadas apresentaram intensificação do vermelho, provavelmente devido à concentração dos pigmentos durante a desidratação das frutas (OSORIO et al., 2007; MASTRANTONIO, PEREIRA e HUBINGER, 2005; MORENO et al., 2000).

Assim como observado para as frutas frescas desidratadas osmoticamente, os resultados sugeriram ter ocorrido retenção dos carotenoides durante a DO dos mamões branqueados.

\subsection{EFEITOS DOS PRÉ-TRATAMENTOS NA SECAGEM CONVECTIVA DO MAMÃO}

Os experimentos de secagem foram conduzidos em dois lotes. No primeiro, foram secas frutas frescas $(F)$ e branqueadas $(B)$, desidratadas osmoticamente (F-DO, B-DO) ou não. Essas amostras foram cobertas com pectina amidada de baixa metoxilação ( $F-C O, B-C O$, F-DO-CO e B-DO-CO) previamente à secagem na segunda bateria de experimentos. Conduziuse a DO das amostras nas condições do ponto central para evitar impregnação excessiva de soluto durante o tratamento osmótico e alcançar diminuição razoável da atividade de água das frutas.

As Tabelas 3 e 4 apresentam a umidade e a atividade de água das amostras secas no primeiro e segundo lotes de experimentos, respectivamente. 
TABELA 3 - UMIDADE E ATIVIDADE DE ÁGUA DAS FRUTAS FRESCAS E BRANQUEADAS,

DESIDRATADAS OSMOTICAMENTE OU NÃO (F, B, F-DO E B-DO) ANTES E APÓS A

SECAGEM CONVECTIVA A $70^{\circ} \mathrm{C}$ POR $240 \mathrm{~min}$

\begin{tabular}{ccccc}
\hline & $\begin{array}{c}\text { Antes da } \\
\text { secagem }\end{array}$ & $\begin{array}{c}\text { Após } \\
\text { a secagem }\end{array}$ & $\begin{array}{c}\text { Antes da } \\
\text { secagem }\end{array}$ & $\begin{array}{c}\text { Após } \\
\text { a secagem }\end{array}$ \\
\hline Amostra & \multicolumn{2}{c}{ Umidade $(\% \mathrm{bu})$} & \multicolumn{2}{c}{ Atividade de água } \\
\hline F & $91,30 \pm 0,04$ & $29,73 \pm 0,003$ & $0,990 \pm 0,001$ & $0,792 \pm 0,004$ \\
B & $93,02 \pm 0,02$ & $7,75 \pm 0,78$ & $0,991 \pm 0,001$ & $0,546 \pm 0,004$ \\
F-DO & $82,46 \pm 0,09$ & $10,30 \pm 0,71$ & $0,976 \pm 0,001$ & $0,481 \pm 0,018$ \\
B-DO & $72,51 \pm 0,05$ & $11,40 \pm 1,13$ & $0,975 \pm 0,000$ & $0,708 \pm 0,004$ \\
\hline
\end{tabular}

Verificou-se ligeira diferença na umidade das frutas frescas utilizadas nos experimentos de secagem entre os dois lotes $(91,30$ e 90,71$)$. O branqueamento e a aplicação da cobertura comestível aumentaram a umidade das amostras devido à imersão em água e/ou solução aquosa. Porém, as atividades de água dessas amostras apresentaram valores similares aos encontrados para os mamões frescos (Tabelas 3 e 4 ).

\section{TABELA 4 - UMIDADE E ATIVIDADE DE ÁGUA DAS FRUTAS FRESCAS E BRANQUEADAS, DESIDRATADAS OSMOTICAMENTE OU NÃO E COBERTAS (F-CO, B-CO, F-DO-CO E B-DO-CO) ANTES E APÓS A SECAGEM CONVECTIVA A 70C POR $240 \mathrm{~min}$}

\begin{tabular}{ccccc}
\hline & $\begin{array}{c}\text { Antes da } \\
\text { secagem }\end{array}$ & $\begin{array}{c}\text { Após a } \\
\text { secagem }\end{array}$ & $\begin{array}{c}\text { Antes da } \\
\text { secagem }\end{array}$ & $\begin{array}{c}\text { Após a } \\
\text { secagem }\end{array}$ \\
\hline Amostra & \multicolumn{2}{c}{ Umidade $(\% \mathrm{bu})$} & \multicolumn{2}{c}{ Atividade de água } \\
\hline F-CO & $91,30 \pm 0,01$ & $30,90 \pm 2,40$ & $0,992 \pm 0,001$ & $0,795 \pm 0,025$ \\
B-CO & $91,92 \pm 0,01$ & $7,35 \pm 0,21$ & $0,991 \pm 0,001$ & $0,497 \pm 0,010$ \\
F-DO-CO & $85,64 \pm 0,04$ & $10,50 \pm 0,01$ & $0,985 \pm 0,001$ & $0,473 \pm 0,010$ \\
B-DO-CO & $76,74 \pm 0,08$ & $10,85 \pm 0,64$ & $0,981 \pm 0,001$ & $0,690 \pm 0,011$ \\
F & $90,71 \pm 0,02$ & & & \\
\hline
\end{tabular}

O tratamento osmótico resultou na diminuição da umidade e da atividade de água das frutas (F-DO, B-DO, F-DO-CO e B-DO-CO) em relação às amostras não desidratadas ( $F$, B, F-CO e $\mathrm{B}-\mathrm{CO})$. As fatias branqueadas apresentaram o menor conteúdo de água após o tratamento osmótico (B-DO e B-DO-CO) (Tabelas 3 e 4), provavelmente devido aos danos causados ao tecido celular da fruta com o tratamento térmico, que aumentaram a incorporação de solutos. Del Valle, Aránguiz e Díaz (1998), Moreno et al. (2000) e Escobar et al. (2007) obtiveram resultados similares trabalhando com maçãs, morangos e cenouras, respectivamente.

Após a secagem, os mamões frescos cobertos ou não (F e F-CO) apresentaram umidade e atividade de água elevadas (Tabelas $3 \mathrm{e} 4$ ). A secagem dessas amostras nas condições estabelecidas $\left(70^{\circ} \mathrm{C}, 1 \mathrm{~m} \cdot \mathrm{s}^{-1}, 240 \mathrm{~min}\right)$ não foi suficiente para reduzir a atividade de água das frutas a valores que garantissem sua segurança microbiológica (<0.6) (YAN, SOUSA-GALLAGHER e OLIVEIRA, 2008).

As frutas frescas desidratadas osmoticamente, cobertas ou não (F-DO e F-DO-CO), apresentaram valores baixos de umidade e as menores atividades de água após a secagem em razão da impregnação de solutos no tecido celular (Tabelas 3 e 4). De fato, essas amostras (F-DO e F-DO-CO) revelaram as menores atividades de água após a secagem dentre todas as amostras, garantindo o não desenvolvimento de micro-organismos nos produtos. 
Após a secagem, as frutas branqueadas cobertas ou não (B e B-CO) evidenciaram as menores umidades dentre todas as amostras pré-tratadas. No entanto, não se verificou o mesmo em relação à atividade de água (apesar de menor que 0,6) dessas amostras (Tabelas 3 e 4).

As frutas branqueadas desidratadas osmoticamente, cobertas ou não (B-DO e B-DO$\mathrm{CO})$, mostraram baixa umidade após a secagem, porém com atividade de água maior do que 0,6. Esse comportamento provavelmente está relacionado às propriedades de sorção de produtos com alto teor de açúcar (Tabelas 3 e 4). Geralmente, as isotermas de sorção de alimentos apresentam formato sigmoidal. Porém, para alimentos com alto teor de compostos solúveis com baixa massa molecular, como os açúcares, o ponto de inflexão está relacionado a baixas umidades e altos valores de atividade de água. Dentre os pré-tratamentos e secagem estudados, o uso do branqueamento precedido pela DO (B-DO e B-DO-CO) foi o menos efetivo na redução da atividade de água.

As Tabelas 5 e 6 apresentam os parâmetros de cor $L^{*}$, $a^{*}$ e $b^{*}$ das amostras secas no primeiro e segundo lotes de experimentos, respectivamente.

O branqueamento causou escurecimento das amostras frescas (Tabelas 5 e 6), fato geralmente observado em frutas e vegetais tratados termicamente (DUTTA, CHAUDHURI e CHAKRABORTY, 2006; OSORIO et al., 2007).

Com relação às frutas frescas, cobertas ou não (F e F-CO), a DO dessas amostras (F-DO e F-DO-CO) aumentou os valores de $a^{*}$ e $b^{*}$ das fatias (Tabelas 5 e 6 ), provavelmente devido à concentração das amostras. ADO das frutas branqueadas (B-DO e B-DO-CO) diminuiu sua claridade e o valor de $b^{*}$ em comparação aos mamões branqueados (B e B-CO). As frutas branqueadas e desidratadas osmoticamente com ou sem adição de cobertura (B-DO e B-DO-CO) resultaram mais escuras do que as frescas (Tabelas 5 e 6 ).

Em trabalhos realizados com abóboras, Dutta, Chaudhuri e Chakraborty (2006) verificaram que o branqueamento causou o escurecimento das amostras e Osorio et al. (2007) observaram diminuição da intensidade da cor de tamarillo após a DO.

Todas as amostras se apresentaram mais escuras após a secagem (Tabelas 5 e 6), com exceção das frutas frescas desidratadas osmoticamente (F-DO) e das branqueadas submetidas à $\mathrm{DO}$ e cobertas (B-DO-CO).

Os parâmetros $a^{*}$ e $b^{*}$ das amostras não cobertas (F, F-DO, B, B-DO) diminuíram com a secagem, sendo o parâmetro $b^{*}$ mais afetado pela secagem do que o $a^{*}$ (Tabela 5). Por outro lado, verificou-se aumento de $a^{*}$ e $b^{*}$ nas amostras cobertas (F-CO, F-DO-CO, B-CO, B-DO-CO) após a secagem, provavelmente porque a cobertura aplicada à superfície das fatias evitou a oxidação dos pigmentos durante a secagem.

Ahmed, Shivhare e Sandhu (2002) relacionaram as mudanças visuais na cor de purê de mamão à degradação dos carotenoides. No presente trabalho, as frutas cobertas apresentaram maiores valores de $a^{*} e b^{*}$ após a secagem (Tabela 6), sugerindo que a aplicação da cobertura de pectina evitou a oxidação dos carotenoides do mamão durante a secagem.

\section{TABELA 5 - L*, $a^{*} E b^{*}$ DAS FRUTAS FRESCAS E BRANQUEADAS, DESIDRATADAS OSMOTICAMENTE OU NÃO (F, B, F-DO E B-DO) ANTES E APÓS A SECAGEM CONVECTIVA A $70^{\circ} \mathrm{C}$ POR $240 \mathrm{~min}$}

\begin{tabular}{ccccccc}
\hline & $\begin{array}{c}\text { Antes da } \\
\text { secagem }\end{array}$ & $\begin{array}{c}\text { Após a } \\
\text { secagem }\end{array}$ & $\begin{array}{c}\text { Antes da } \\
\text { secagem }\end{array}$ & $\begin{array}{c}\text { Após a } \\
\text { secagem }\end{array}$ & $\begin{array}{c}\text { Antes da } \\
\text { secagem }\end{array}$ & $\begin{array}{c}\text { Após a } \\
\text { secagem }\end{array}$ \\
\hline \multicolumn{2}{c}{$\mathrm{L}^{*}$} & \multicolumn{2}{c}{$\mathrm{a}^{*}$} & $\mathrm{~b}^{*}$ & \\
\hline F & $55,93 \pm 0,41$ & $45,91 \pm 2,11$ & $31,26 \pm 2,37$ & $29,67 \pm 1,70$ & $43,69 \pm 1,62$ & $28,06 \pm 1,09$ \\
B & $44,78 \pm 0,30$ & $43,82 \pm 0,42$ & $29,22 \pm 0,32$ & $27,77 \pm 1,52$ & $44,78 \pm 0,14$ & $37,12 \pm 2,07$ \\
F-DO & $55,48 \pm 1,05$ & $57,59 \pm 2,68$ & $34,34 \pm 1,11$ & $29,64 \pm 1,40$ & $48,25 \pm 0,29$ & $45,25 \pm 1,64$ \\
B-DO & $43,08 \pm 0,74$ & $40,24 \pm 0,38$ & $31,95 \pm 0,72$ & $29,26 \pm 0,79$ & $44,23 \pm 0,87$ & $24,17 \pm 1,04$ \\
\hline
\end{tabular}




\section{TABELA 6 - L*, $a^{*} E b^{*}$ DAS FRUTAS FRESCAS E BRANQUEADAS, DESIDRATADAS OSMOTICAMENTE OU NÃO E COBERTAS (F-CO, B-CO, F-DO-CO E B-DO-CO) ANTES E APÓS A SECAGEM CONVECTIVA A $70^{\circ} \mathrm{C}$ POR $240 \mathrm{~min}$}

\begin{tabular}{ccccccc}
\hline & $\begin{array}{c}\text { Antes da } \\
\text { secagem }\end{array}$ & $\begin{array}{c}\text { Após a } \\
\text { secagem }\end{array}$ & $\begin{array}{c}\text { Antes da } \\
\text { secagem }\end{array}$ & $\begin{array}{c}\text { Após a } \\
\text { secagem }\end{array}$ & $\begin{array}{c}\text { Antes da } \\
\text { secagem }\end{array}$ & $\begin{array}{c}\text { Após a } \\
\text { secagem }\end{array}$ \\
\hline & \multicolumn{2}{c}{$\mathrm{L}^{*}$} & & $\mathrm{a}^{*}$ & $\mathrm{~b}^{*}$ & \\
\hline F-CO & $47,09 \pm 1,08$ & $39,03 \pm 0,94$ & $24,77 \pm 2,02$ & $31,55 \pm 0,6$ & $37,99 \pm 3,43$ & $40,43 \pm 2,55$ \\
B-CO & $44,18 \pm 0,70$ & $42,04 \pm 2,04$ & $26,68 \pm 0,76$ & $35,50 \pm 0,46$ & $31,25 \pm 0,88$ & $47,14 \pm 1,16$ \\
F-DO-CO & $57,40 \pm 2,55$ & $51,61 \pm 1,00$ & $28,68 \pm 2,40$ & $33,09 \pm 0,53$ & $43,04 \pm 0,43$ & $45,77 \pm 0,86$ \\
B-DO-CO & $43,29 \pm 0,42$ & $54,87 \pm 0,75$ & $26,96 \pm 0,15$ & $35,16 \pm 0,71$ & $26,62 \pm 1,28$ & $45,41 \pm 0,87$ \\
\hline
\end{tabular}

\section{CONCLUSÃO}

A DO utilizando diferentes tempos de desidratação e concentrações da solução osmótica apresentou pequenas e não significativas alterações na cor de amostras de mamão nas faixas estudadas, mostrando a preservação dos pigmentos do mamão durante o processamento. Uma expressão para a atividade de água das amostras branqueadas desidratadas osmoticamente foi encontrada.

Os experimentos de secagem mostraram que o branqueamento não foi apropriado para preservar a cor das amostras, enquanto que o branqueamento precedido pela DO não foi efetivo na redução da atividade de água das frutas.

A DO das frutas frescas cobertas ou não resultaram nas amostras com as menores atividades de água após a secagem, evidenciando o potencial desse processamento na obtenção de produtos estáveis com economia de energia.

As frutas cobertas apresentaram valores maiores de $a^{*} e b^{*}$ do que as não cobertas após a secagem, sugerindo que a cobertura evitou a oxidação dos pigmentos coloridos durante a secagem.

\section{ABSTRACT}

\section{COMBINED METHODS TO OBTAIN DRIED PAPAYA OF FORMOSA CULTIVAR (Carica papaya)}

The aim of this work was to evaluate the influence of blanching in hot water, osmotic dehydration in sucrose solution and pectin coating on color and water activity of papaya slices after convective drying. On the first experiments, the influence of concentration and time of dehydration on color changes and water activity of fresh and blanched papaya slices was evaluated based on a factorial design. It was not verified significant color changes at the studied ranges. An expression for the water activity of osmotically dehydrated blanched samples was found. Then, the influences of blanching, osmotic dehydration, edible coating and combinations among these pre-treatments on color and water activity were evaluated after convective drying of papaya slices. Blanching was not an advantageous pre-treatment to maintain color and reduce water activity of dried fruits. Osmotically dehydrated fresh samples with or without coating presented the best results.

KEY-WORDS: OSMOTIC DEHYDRATION; BLANCHING; EDIBLE FILMS; DRYING; PAPAYA.

\section{REFERÊNCIAS}

1 AHMED, J.; SHIVHARE, U.S.; SANDHU, K.S. Thermal degradation kinetics of carotenoids and visual color of papaya puree. Food Engineering and Physical Properties, v.67, n.7, p.2692-2695, 2002. 
2 ALZAMORA, S.M.; GERSCHENSON, L.N.; VIDALES, S.L.; NIETO, A. Structural changes in the minimal processing of fruits: some effects of blanching and sugar impregnation. In: FOOD Engineering 2000. New York: Chapman and Hall, 1997. p. 117-139.

3 BARROS NETO, Benício de; SCARMINIO, Ieda Spacino; BRUNS, Roy Edward. Como fazer experimentos: pesquisa e desenvolvimento na ciência e na indústria. Campinas: Editora da UNICAMP, 2002.

4 CHAN J.R.; KWOK, S.C.M. Importance of enzyme inactivation prior to extraction of sugars from papaya. Journal of Food Science, v.40, p.770-771, 1975

5 CUQ, B.; GINTARD, N.; GUILBERT, S. Edible films and coatings as active layers. In: EDIBLE active layers. London: Blackie Academic \& Professional, 1995

6 DEL VALLE, J.M.; ARÁNGUIZ, V.; DÍAZ, L. Volumetric procedure to assess infiltration kinetics and porosity of fruits by applying a vacuum pulse. Journal of Food Engineering, v.38, p.207-221, 1998.

7 DOYMAZ, I. The kinetics of forced convective air-drying of pumpkin slices. Journal of Food Engineering, v.79, p.243248,2007

8 DUTTA, D.; CHAUDHURI, U.R.; CHAKRABORTY, R. Effect of thermal treatment on the $\beta$-carotene content, color and textural properties of pumpkin. Journal of Food Science and Technology, v.3, p.607-611, 2006.

9 EREN, I.; KAYMAK-ERTEKIN, F. Optimization of osmotic dehydration of potato using response surface methodology. Journal of Food Engineering, v.79, p.344-352, 2007.

10 ESCOBAR, M.P.; GALINDO, F.G.; WADSÖ, L.; NÁJERA, J.R.; SJÖHOLM, I. Effect of long-term storage and blanching pre-treatment on the osmotic dehydration kinetics of carrots (Daucus carota L. cv. Nerac). Journal of Food Engineering, v.81, p.313-317, 2007

11 FERNANDES, F.A.N.; RODRIGUES, S.; GASPARETO, O.C.P.; OLIVEIRA, E.L. Optimization of osmotic dehydration of papaya followed by air-drying. Food Research International, v.39, p.492-498, 2006.

12 FITO, P.; CHIRALT, A.; BETORET, N.; CHÁFER, M.J. Vaccum impregnation and osmotic dehydration in matrix engineering: application and functional fresh food development. Journal of Food Engineering, v.49, p.175-183, 2001.

13 GARCIA, C.C.; MAURO, M.A.; KIMURA, M. Kinetics of osmotic dehydration and air-drying of pumpkins (Cucurbita moschata). Journal of Food Engineering, v.82, p.284-291, 2007.

14 HENG, H.; GUILBERT, S.; CUQ, J.L. Osmotic dehydration of papaya: influence of process variables on the product quality. Sciences des Aliments, v.10, p.831-848, 1990.

15 HEREDIA, A.; BARRERA, C.; ANDRÉS, A. Drying of cherry tomato by a combination of different dehydration techniques. Comparison of kinetics and other related properties. Journal of Food Engineering, v.80, p.111-118, 2007.

16 HUNTERLAB INC. ColorFlex user's guide. Manual Version 2.5. Reston, VA, USA, 2007. 188 p.

17 KIMURA, M.; RODRIGUEZ-AMAYA, D.B.; YOKOYAMA, S.M. Cultivar differences and geographic effects on the carotenoid composition and vitamin A value of papaya. LWT, v.24, p.415-418, 1991

18 KOWALSKA, A.; LENART, A.; LESZCZYK, D. The effect of blanching and freezing on osmotic dehydration of pumpkin. Journal of Food Engineering, v.86, p.30-38, 2008.

19 LEERATANARAK, N.; DEVAHASTIN, S.; CHIEWCHAN, N. Drying kinetics and quality of potato chips undergoing different drying techniques. Journal of Food Engineering, v.77, p.635-643, 2006.

20 LUZURIAGA, D.A.; BALABAN, M.O.; YERALAN, S. Analysis of visual quality attributes of white shrimp by machine vision. Journal of Food Science, v.62, p.113-118, 1997.

21 MASTRANTONIO, S.D.S.; PEREIRA, L.M.; HUBINGER, M.D. Osmotic dehydration kinetics of guavas in maltose solutions with calcium salt. Alimentos e Nutrição, v.16, p.309-314, 2005.

22 MORENO, J.; CHIRALT, A.; ESCRICHE, I.; SERRA, J.A. Effect of blanching/osmotic dehydration combined methods on quality and stability of minimally processed strawberries. Food Research International, v.33, p.609-616, 2000.

23 NOVASINA AG. AW sprint TH 500: operating instructions. Lanchen, Switzerland, 1999. 86 p.

24 OSORIO, C.; FRANCO, M.S.; CASTAÑO, M.P.; GONZÁLEZ-MIRET, M.L.; HEREDIA, F.J.; MORALES, A.L. Colour and flavour changes during osmotic dehydration of fruits. Innovative Food Science and Emerging Technologies, v.8, p.353-359, 2007

25 RODRIGUES, A.C.C.; CUNHA, R.L.; HUBINGER, M.D. Rheological properties and color evaluation of papaya during osmotic dehydration processing. Journal of Food Engineering, v.59, p.129-135, 2003. 
26 SERENO, A.M.; MOREIRA, R.; MARTINEZ, E. Mass transfer coefficients during osmotic dehydration of apple in single and combined aqueous solutions of sugar and salt. Journal of Food Engineering, v.47, p.43-49, 2001.

27 SHI, J.; LE MAGUER, M.; KAKUDA, Y.; LIPTAY, A.; NIEKAMP, F. Lycopene degradation and isomerization in tomato dehydration. Food Research International, v.32, p.15-21, 1999.

28 STATSOFT INC. Statistica. Version 7.0. Tulsa, USA, 2004.

29 TONON, R.V.; BARONI, A.F.; HUBINGER, M.D. Osmotic dehydration of tomato in ternary solutions: influence of process variables on mass transfer kinetics and an evaluation of the retention of carotenoids. Journal of Food Engineering, v.82, p.509-517, 2007.

30 TORREGIANI, D.; BERTOLO, G. Osmotic pre-treatments in fruit processing: chemical, physical and structural effects. Journal of Food Engineering, v.49, p.247-253, 2001.

31 VARGAS, M.; PASTOR, C.; CHIRALT, A.; McCLEMENTS, D.J.; GONZÁLEZ-MARTíNEZ, C. Recent advances in edible coatings for fresh and minimally processed fruits. Critical Reviews in Food Science and Nutrition, v.48, p.496-511, 2008

32 YAN, Z.; SOUSA-GALLAGHER, M.J.; OLIVEIRA, F.A.R. Sorption isotherms and moisture sorption hysteresis of intermediate moisture content banana. Journal of Food Engineering, v.86, p.342-348, 2008.

33 ZHAO, Y.P.; CHANG, K.C. Sulfite and starch affect color and carotenoids of dehydrated carrots (Daucus carota) during storage. Journal of Food Science, v.60, p.324-347, 1995.

\section{AGRADECIMENTOS}

Os autores agradecem à CAPES pela bolsa de estudos, à FAPESP pelo suporte financeiro (Proc 07/07586-0 e Proc 09/00519-0), à PURAC Synthesis (Brasil) e à Danisco (Brasil). 